Service social

\title{
Une intervention de groupe d'orientation féministe en santé mentale
}

\section{Suzanne Rondeau}

Volume 43, numéro 3, 1994

Intervention individualisée et empowerment

URI : https://id.erudit.org/iderudit/706672ar

DOI : https://doi.org/10.7202/706672ar

Aller au sommaire du numéro

Éditeur(s)

École de service social de l'Université Laval

ISSN

1708-1734 (numérique)

Découvrir la revue

Citer cet article

Rondeau, S. (1994). Une intervention de groupe d'orientation féministe en santé mentale. Service social, 43(3), 123-137. https://doi.org/10.7202/706672ar
Résumé de l'article

Partant de la conviction que les intervenants sociaux transposent trop rarement dans l'écrit la richesse de leurs expériences de pratique, l'auteure décrit les principales caractéristiques d'une expérience de groupe d'orientation féministe en santé mentale. Elle expose d'abord la problématique de départ et précise les fondements théoriques qui servent d'appui au modèle sélectionné. Elle met ensuite en lumière les diverses composantes du programme et de sa réalisation au fil des ans. Les résultats obtenus sont liés à des typologies reconnues dont l'une concerne les effets sur les sentiments, les comportements et les attitudes. L'expérience est enfin interprétée comme rejoignant d'importantes stratégies de prévention sociale en santé mentale. 


\title{
Une intervention de groupe d'orientation féministe en santé mentale
}

\author{
Suzanne RONDEAU \\ Travailleuse sociale \\ Département de psychiatrie \\ Centre hospitalier de I'Université Laval (CHUL)
}

Dans les échanges de vues entre intervenantes et intervenants sociaux, les questions d'identité professionnelle, de statut de la profession se révèlent encore souvent très présentes. La fréquentation de réunions en milieu de travail et les rassemblements de praticiens et praticiennes lors de colloques et congrès nous ramènent pourtant fréquemment à l'évidence de réalisations particulières remarquables par leur qualité. Engagés dans un domaine d'action, serions-nous peu portés à traduire en mots les gestes de notre quotidien ? Nous prétextons souvent la lourdeur de la tâche, I'absence de moyens mis à notre disposition par nos organismes employeurs, ce qui est au départ indéniable. Ne sommes-nous pas par ailleurs porteurs d'une vision de l'écriture comme étant la chasse gardée d'une élite intellectuelle en mesure $d^{\prime}$ illustrer des découvertes exceptionnelles ?

$C^{\prime}$ est dans un état d'esprit axé sur la pertinence d'une mise en valeur de nos expériences cliniques qu'a été conçu cet article. 
Un travail de longue date en milieu psychiatrique, de même que la réalisation d'études de maîtrise en service social, avait stimulé chez moi l'intérêt d'élaborer un programme de groupe plus spécifiquement destiné à la clientèle féminine toujours majoritaire en consultation psychiatrique. C'est en collaboration étroite avec une collègue travailleuse sociale, également étudiante à la maîtrise, que les diverses étapes du projet ont été réalisées: préparation théorique et pratique, présentation du programme en milieu de travail et mise en œuvre à partir du printemps de 1985. À la suite de l'expérimentation durant plusieurs années de ce programme, j'ai été amenée à me poser diverses questions auxquelles je tenterai de répondre. En quoi le modèle privilégié, à savoir une intervention de groupe d'orientation féministe, correspond-il aux besoins réels de la population concernée, en rapport avec ses fondements théoriques? Que peuvent révéler les résultats colligés? Quelles sont les limites de cette expérience? Sans avoir été l'objet de recherches systématiques, I'exercice approfondit une expérience documentée par des données cliniques conservées au fil des ans et alimentée de réflexions quant à leur correspondance au modèle théorique. Après avoir exposé la problématique et les fondements théoriques du modèle privilégié, je décrirai brièvement le programme mis en œuvre et sa réalisation. Certains résultats de I'intervention seront ensuite mis en évidence auxquels nous apporterons notre interprétation.

\section{LA PROBLÉMATIQUE}

Notre questionnement relevait directement des interrogations du mouvement féministe des années 60 et surtout 70, particulièrement celles portant sur la santé mentale des femmes. Par l'intermédiaire de diverses études et enquêtes de l'époque, le système de soins était ouvertement remis en question : stéréotypes sexistes chez les intervenants et intervenantes en santé mentale (Broverman, 1970; Sherman et al., 1978), surconsommation de psychotropes par les femmes (Cooperstock, 1980) ou encore ignorance des origines sociales de la maladie mentale. Les facteurs socioculturels liés à cet aspect ont d'ailleurs été mis en évidence par plusieurs auteures féministes, telles Sturdivant (1980), Corbeil et al. (1983), Wyckoff (1980), etc. Une recherche effectuée à l'époque dans notre milieu de travail (Brochu et Brousseau-Gingras, 1984), portant sur la connaissance de la clientèle service social de notre clinique externe de psychiatrie ainsi que des services offerts, concluait à l'évidence d'une clientèle en majorité 
féminine. Des services lui étaient fournis sous un mode strictement individuel tant par les travailleuses sociales que par les autres intervenants et intervenantes en place.

Les femmes qui consultent en psychiatrie expriment leur détresse par le moyen de divers symptômes, tels les éléments dépressifs, les troubles anxieux souvent de type phobique, les troubles psychosomatiques, les troubles de personnalité et les troubles du comportement alimentaire. Quelles que soient les difficultés présentées, le fonctionnement personnel et social est bien souvent perturbé. Une faible estime de soi, un sentiment de marginalité et d'inutilité, de même que I'isolement social, reviennent constamment dans les propos exprimés. Dans une perspective féministe, ces appels à l'aide ne sont pas considérés comme étant d'ordre psychopathologique, mais plutôt liés aux divers conditionnements socioculturels auxquels sont soumises les femmes. À partir de la nomenclature de Sturdivant (1980: 149), on pourrait les résumer de la façon qui suit. Les symptômes sont parfois le résultat du conditionnement au rôle féminin : centration sur le rôle d'épouse et de mère. Ils peuvent également résulter de la socialisation féminine par le conditionnement aux qualités dites féminines et à l'évitement de comportements dits masculins, comme l'expression de l'agressivité. Ils peuvent enfin représenter pour les femmes des tactiques de survie en réponse au manque de pouvoir sur leur propre vie: ainsi les troubles hystériques, les troubles passifs agressifs ou autres deviendraient des réactions à cette absence d'emprise sur leur destin personnel.

\section{LES FONDEMENTS THÉORIQUES DU MODÈLE}

\section{L'intervention féministe}

L'intervention féministe apparaissait dès le départ être l'approche la plus susceptible de répondre aux besoins exprimés par ces femmes qui consultent. Dans cette perspective, la compréhension des problèmes présentés ne se limite pas aux aspects biologiques et psychologiques du vécu des femmes, mais elle englobe les facteurs culturels, sociaux, politiques et économiques qui ont autant d'impact sur leur vie. Avant d'expliquer en quoi cette vision globale est devenue un des fondements majeurs de notre intervention, permettons-nous un retour historique sur cette quête de libération des femmes par la recherche d'une identité et d'une expression d'elles-mêmes qui soit authentique. 
Les difficultés qu'éprouvent les femmes qui consultent en santé mentale ne nous semblent pas étrangères aux luttes des femmes de ce siècle et du siècle dernier pour parvenir à s'imposer dans la vie publique. Aussi nous apparaît-il intéressant à ce point de faire référence à la littérature féminine du dix-neuvième siècle. Afin de pouvoir être reconnues comme écrivaines, les femmes de cette époque ont dû vivre dans le secret, dans la clandestinité. Ce fut le sort de la romancière anglaise Jane Austen qui, dans le salon familial, prenait paraît-il grand soin de cacher ses manuscrits, afin que ni les domestiques, ni les visiteurs ne soient en mesure de soupçonner son travail. Dans la même ligne de pensée, on peut aussi se demander pourquoi Aurore Dupin jugea bon de changer son nom en celui de George Sand ou encore pourquoi fut-elle tant la cible de railleries et de qualificatifs outrageux, tel celui $\mathrm{d}^{\prime}$ " hommasse»? Sans aucun doute, ces écrivaines souffraient de l'étroitesse de vie qui leur était imposée: à l'époque, les femmes ne circulaient pas seules, ne fréquentaient pas les restaurants, ne voyageaient que rarement... Dans Jane Eyre, Charlotte Brontë (Woolf, 1929: 90) illustre bien à son tour les frustrations associées à ce vécu :

Elles (les filles) ont autant que leurs frères besoin d'exercice pour leurs facultés et d'un terrain pour leurs efforts ; [....] C'est étourderie que de les condamner ou de se moquer d'elles si elles cherchent à faire davantage ou à apprendre plus que ce que la coutume a décrété nécessaire à leur sexe.

Pas étonnant non plus que, malgré leurs chefs-d'œuvre, ces femmes n'aient pu bien souvent donner la pleine mesure de leur génie: non seulement écrivaient-elles dans le secret et malgré le tumulte des activités quotidiennes imposées, mais elles s'exprimaient dans la peur et la rage. D'où les conclusions d'auteures célèbres du début de la première partie du vingtième siècle, comme Virginia Woolf dans Une chambre à soi: "Il est indispensable qu'une femme possède quelque argent et une chambre à soi si elle veut écrire une œuvre de fiction » (Woolf, 1929: 6). Ou, vingt ans plus tard, celles de Simone de Beauvoir dans Le deuxième sexe: "Les restrictions que l'éducation et la coutume imposent à la femme limitent sa prise sur l'univers " (De Beauvoir, 1949: 476), puis: "Les contraintes dont elle est entourée et toute la tradition qui pèse sur elle empêchent qu'elle ne se sente responsable de l'univers»(De Beauvoir, 1949: 478).

En cette seconde moitié du vingtième siècle, vu les acquis découlant des «années chaudes du féminisme » des années 70 , on tend souvent à croire ou à laisser croire que le conditionnement féminin et ses conséquences sont choses du passé. L'accès à la vie 
publique s'est certes amélioré sous divers aspects : accès à l'instruction, à l'emploi, à la vie politique, etc. Cependant, l'omniprésence du conditionnement social orienté vers le service aux autres, vers l'adoption de comportements stéréotypés demeure subtilement perceptible. Ne retrouve-t-on pas en effet l'exacerbation de cet aspect au cœur des symptômes que présentent les femmes qui consultent pour des problèmes de santé mentale? Combien de fois les symptômes dépressifs, anxieux ou psychosomatiques ne recouvrent-ils pas, si nous savons les analyser, une colère interdite aux femmes depuis longtemps, une culpabilité de ne pas être conformes ou encore une incapacité de leur part de croire en leur droit de se définir ou de se réaliser pleinement?

$C^{\prime}$ est dans cette perspective que semble pertinent notre choix d'une intervention féministe qui, à la base, tient compte du rôle des facteurs socioculturels dans la genèse de la détresse affective chez les femmes. Selon certaines auteures féministes associées à la relation d'aide, dont Carol Gilligan (1982), les femmes ont été définies principalement par leur rapport à autrui, avec un accent mis sur la notion du "faire plaisir » plutôt que de se faire plaisir et de prendre soin de soi (Gilligan, 1982: 16, 17). Il importe donc, selon Sturdivant, de les aider à trouver "la liberté et la capacité de se définir ellesmêmes et de déterminer leurs propres besoins, valeurs, actions, pensées, même si dans le passé elles ont souvent abandonné à d'autres cette capacité »(Sturdivant, 1980: 124).

Ainsi, à l'intérieur de notre programme, les thèmes tels que I'estime de soi, I'affirmation de soi, la dépendance affective et la santé mentale sont-ils principalement orientés vers ce but. L'interprétation des symptômes telle qu'exposée précédemment semble également rejoindre la réalité des femmes. Les participantes apprennent enfin à reconnaître des facteurs d'influence plus rarement décelés par leur entourage ou même par leurs intervenants ou intervenantes. Elles prennent conscience de la multiplicité des facteurs de causalité influant sur leurs situations de vie. Elles constatent que leurs difficultés sont aussi le lot de plusieurs autres femmes de leur société. Dans une telle perspective, elles en viennent à considérer la violence conjugale comme liée aux rapports de pouvoir hommes-femmes, la pauvreté féminine en lien avec le faible taux d'instruction des femmes et leur insertion dans des métiers féminins sous-payés, avec le cumul des tâches domestiques trop souvent dévolues aux femmes, etc. Le "privé» devient alors "politique» et le changement personnel apparaît compatible avec le changement social. 
Notre intervention ne se limite pas à la prise de conscience de ces aspects que Mary Russell (1984) nomme "analyse sociale» et Susan Sturdivant (1980) «conscience féministe». Elle est également orientée vers l'action, car les participantes, en plus de travailler à développer certaines croyances (droit à l'affirmation, droit au plaisir, droit de se réaliser, etc.), font l'apprentissage d'habiletés sociales (communication, affirmation de soi, socialisation), de l'entraide et de l'aide mutuelle, avec la possibilité d'un transfert de ces acquis dans leur mode de vie.

\section{L'intervention de groupe}

Certaines auteures féministes, dont Cardin et Home (1983), tendent à croire que le fait que l'approche individuelle soit encore le modèle privilégié par la majorité des intervenants et intervenantes maintient les femmes dans la dépendance et l'isolement contre lesquels elles doivent justement se défendre. En accord avec les objectifs qu'elle poursuit, l'approche féministe donne pour sa part une place centrale à l'intervention de groupe. En effet, celle-ci favorise l'échange de vues entre pairs à partir de problèmes communs, l'apprentissage d'habiletés, la resocialisation, la pratique de l'entraide et la lutte contre l'isolement. Notre choix de ce type d'intervention découle évidemment de l'importance que nous accordons à ces facteurs en rapport avec la démarche proposée.

\section{LA DESCRIPTION DU PROGRAMME}

\section{Les objectifs et le déroulement du programme}

Le programme est offert à la clinique externe de psychiatrie d'un hôpital général. Toutes les catégories de professionnels et professionnelles peuvent y adresser des femmes, à savoir les médecins, les travailleuses sociales, les infirmières et infirmiers et la psychologue. Le personnel de l'Unité de soins psychiatriques y a également accès, principalement pour l'orientation d'usagères qui envisagent une sortie imminente de l'hôpital. Les critères de sélection sont souples: avoir entre 30 et 55 ans, posséder une capacité minimale de fonctionner en groupe et de se fixer des buts individuels et, enfin, être motivée à participer à l'expérience. D'où la place importante accordée à celle qui consulte, devant sa décision de s'engager dans l'intervention 
proposée. Seules les femmes qui vivent un état de crise aiguë nécessitant plutôt une intervention individuelle sont généralement exclues.

L'objectif général de l'intervention vise le changement personnel, c'est-à-dire la résolution des problèmes pour lesquels les membres consultent, au moyen d'un travail centré sur l'estime et l'affirmation de soi. Les objectifs secondaires de conscientisation, d'entraide et de socialisation favorisent le développement personnel des membres et celui du groupe comme tel.

Stabilisé à 13 rencontres depuis le printemps 1990, le programme a varié entre 11 et 14 soirées d'une durée de deux heures et demie chacune, y compris une relance trois mois après la fin de l'intervention. C'est, il va sans dire, un programme à court terme, structuré du fait de l'utilisation de thèmes préétablis, mais qui offre de la souplesse dans son fonctionnement: insertion $d^{\prime} u n$ "retour sur la semaine» à I'horaire de chaque rencontre permettant d'aborder des préoccupations hors thème, distribution d'une pochette de documents de lecture libre, information sur les ressources communautaires, retour sur la rencontre hebdomadaire, etc. Les thèmes proposés ont un lien direct avec I'intervention féministe car, à travers ces thèmes, on trouve, sous-jacente, une conscientisation aux facteurs socioculturels à l'origine des difficultés éprouvées. La sélection des thèmes a varié selon les époques par suite des commentaires des participantes lors des bilans et de I'analyse des animatrices. Actuellement, l'accent est mis sur l'estime de soi, l'affirmation de soi, la santé mentale et la dépendance affective qui occupent chacune deux rencontres. La culpabilité constitue également un thème complémentaire, alors que, par le passé, les thèmes de la solitude, de la sexualité, de la violence et des rôles sociaux selon le sexe étaient au programme. Une soirée est également consacrée à la détermination d'un objectif individuel en raison de l'exigence que notre programme requiert, à savoir que les membres du groupe se fixent un objectif individuel concret et mesurable, réalisable tout au moins partiellement durant l'intervention. Les participantes doivent partager cet objectif avec le groupe, afin de favoriser le soutien mutuel, et en faire l'évaluation à la dernière rencontre.

Une analyse des choix d'objectifs individuels effectués amène à constater que la majorité de ceux-ci portent sur l'estime et l'affirmation de soi, alors que d'autres renvoient plutôt à la connaissance de soi et à l'organisation d'un mode de vie plus épanouissant. Le tableau qui suit constitue un résumé presque exhaustif de l'ensemble des choix habituels des participantes aux sessions de groupe. 
Tableau sommaire des objectifs individuels

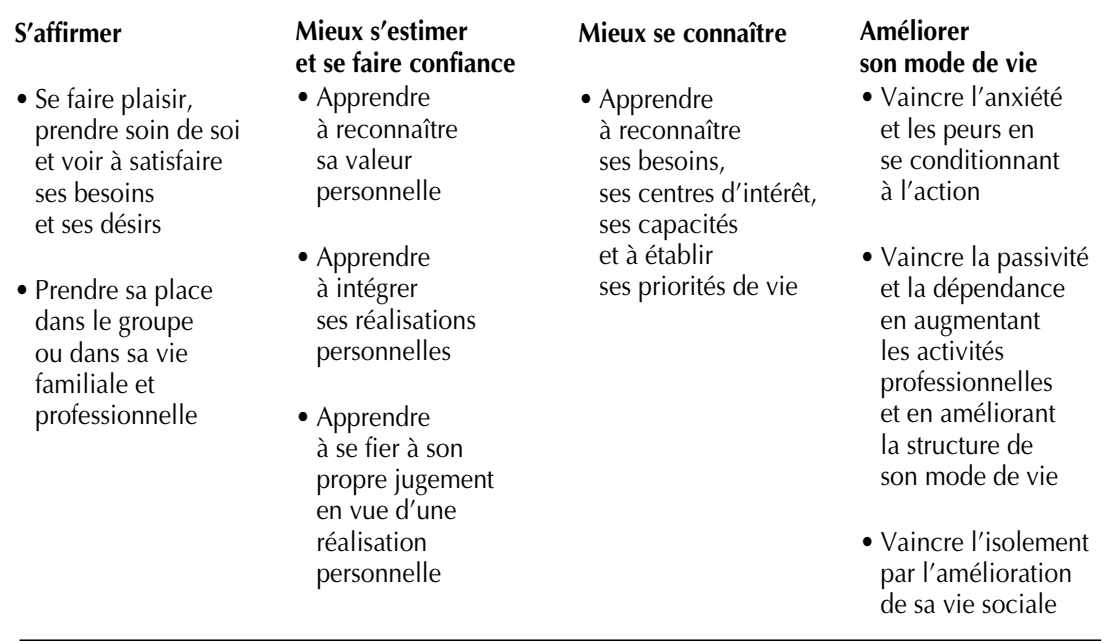

L'intervention, faite en coanimation avec une travailleuse sociale du milieu, inclut un partage des tâches à toutes les étapes du déroulement du groupe, de même qu'un idéal commun d'offrir une collaboration caractérisée par la coopération plutôt que par la compétition. Nous adhérons au principe féministe de la relation égalitaire en termes de démythification des thérapeutes, de partage du savoir et de la croyance en la primauté de la cliente comme experte vis-à-vis de ses sentiments, valeurs et choix de vie. Comme rôles, nous privilégions celui de facilitatrices dans l'animation. Nous sommes également par moments enseignantes eu égard, par exemple, à I'habileté de l'affirmation. Le rôle de thérapeutes nous permet enfin de donner au besoin de l'information et de favoriser l'utilisation de notre expérience clinique par l'emploi de certaines techniques d'intervention féministe décrites par Mary Russell (1984).

\section{La réalisation du programme}

Afin de compléter le tableau descriptif de l'intervention, il convient de relever certaines données statistiques susceptibles d'illustrer l'ampleur du projet réalisé, de même que sa stabilité de fonctionnement. Entre le printemps 1985 et le printemps 1994, dix-neuf groupes ont été tenus, c'est-à-dire que le programme a été réalisé de façon régulière à raison de deux sessions par année. Selon les statistiques 
compilées, près de 140 femmes I'ont suivi, soit une moyenne de huit participantes par groupe. Sont exclues de ce nombre les femmes qui n'ont participé qu'à une ou deux rencontres, de même que la proportion plus infime de celles qui ont abandonné l'intervention après quelques rencontres. Quant à la règle d'assiduité, présentée comme partie intégrante de l'expérience parce qu'elle favorise un engagement dans le groupe, elle est, de façon générale, minutieusement respectée, les participantes ne s'absentant que pour des raisons relevant de leur état de santé ou d'obstacles dans leur vie familiale ou professionnelle.

\section{LES RÉSULTATS OBSERVÉS}

Ainsi qu'il est indiqué en introduction, notre observation des résultats ne relève pas d'une utilisation systématique d'instruments d'évaluation à divers moments de l'expérience. Elle découle d'abord de la philosophie féministe qui postule l'absence d'un corps immuable de "vérités » sur la nature et le comportement humain qui s'appliquerait universellement dans le traitement de la détresse psychologique. La thérapie féministe est alors perçue, comme chez Sturdivant, tel

un processus dans lequel la philosophie, les théories et les techniques de traitement sont constamment influencées par la culture, aussi bien qu'en perpétuelle transformation, pour rencontrer les besoins et les demandes du milieu social (Sturdivant, $1980: 219$ ).

En même temps que nous considérons le contenu des comptes rendus bilans comme significatif, nous tenons pour acquis que, de façon concomitante à l'intervention, de nombreux autres facteurs ont pu contribuer à l'amélioration ou à la détérioration du bien-être de la clientèle visée, tels que la médication, l'utilisation d'autres formes d'aide, la mouvance des circonstances de vie, etc.

Une lecture attentive des comptes rendus verbaux et écrits des rencontres bilans des 19 groupes réalisés nous a cependant amenée à considérer avec une attention particulière une typologie de résultats connue en intervention de groupe de femmes, soit celle de Home (1988). Dans la revue Service social, celle-ci fait référence à trois niveaux de changements qu'il est possible d'observer dans les groupes de ce type, soit ceux des sentiments, des comportements et des attitudes. Nous tenterons d'établir certaines correspondances entre nos résultats et cette typologie. 


\section{Le niveau des sentiments}

Beaucoup de femmes modifient leurs sentiments à l'égard d'elles-mêmes et se sentent plus confiantes, moins coupables, plus solides dans leur identité et moins dépendantes des relations avec les hommes et les enfants (Home, 1988: 65).

Ce résultat constaté par Home se retrouve en effet dans les bilans écrits des femmes qui ont participé à nos groupes. Ainsi peuton y lire par rapport à la confiance en soi et en l'avenir: « Ce groupe $\mathrm{m}^{\prime}$ a aidée à reprendre espoir, mais aussi à développer le goût de prendre des risques. »En référence à l'identité, on entend: «Ce groupe $\mathrm{m}^{\prime}$ a aidée à mieux découvrir qui je suis, quelles capacités j'ai. » L'aspect de la normalisation par l'intervention de groupe est omniprésent: "Je ne me sens plus seule à vivre des problèmes. » La culpabilité s'avère bien souvent atténuée: "Je vois les choses différemment, je porte moins sur moi la responsabilité de ma famille, de I'univers entier comme autrefois. » La dépendance à l'égard des autres est également touchée: "Je comprends mieux mes besoins comme personne, je suis plus consciente que j'ai aussi des droits à respecter et à faire respecter. »Ces propos ne sont pas le lot d'exceptions, mais reviennent couramment lors des rencontres bilans.

\section{Le niveau des comportements}

Sur le plan des changements comportementaux, les acquis les plus probants se retrouvent dans I'adoption de comportements plus affirmatifs et plus autonomes. L'accent placé dans notre programme sur l'apprentissage de l'affirmation en vue de contrer une socialisation trop souvent axée sur la passivité, le refoulement de l'agressivité et le renoncement à une réalisation personnelle semble répondre à un besoin réel des participantes. En effet, bien avant que ne s'effectue le travail sur ce thème (cinquième et sixième rencontres), les femmes expriment leur insatisfaction par rapport à elles-mêmes sous cet aspect et, dès la deuxième rencontre, elles choisissent fréquemment un objectif individuel qui y est relié. Au moment des bilans, plusieurs participantes mentionnent avoir réussi à acquérir des comportements plus affirmatifs dans diverses aires de leur vie: vis-à-vis des membres de leur réseau primaire (conjoint, enfants, parenté, voisins, amis et amies), dans leur vie professionnelle (patrons ou patronnes, compagnons et compagnes de travail, etc.) ou dans leur vie en général (rapports avec les divers services utilisés, tels les services de santé, de consommation, etc.). L'acquisition de comportements plus auto- 
nomes, par ailleurs souvent reliés à la capacité d'affirmation, se trouve également fréquemment rapportée : adoption de changements dans l'organisation familiale, relevé $d^{\prime}$ actions faites en vue de modifications dans la vie professionnelle, telles qu'un retour aux études ou une entrée sur le marché du travail ou du bénévolat, réaménagements effectués sur le plan des loisirs et de la vie sociale, etc. En accomplissant ces gestes, les participantes expriment leur sensation $d^{\prime}$ avoir plus de contrôle et de pouvoir sur leur vie, plus de prise sur leur environnement.

\section{Le niveau des attitudes}

Le troisième type de changements relevés par Home correspond aux changements dans les attitudes par rapport aux rôles féminins. Ce type de changements est typique des groupes $d^{\prime}$ orientation féministe. Au moment des rencontres bilans, une certaine proportion des femmes constatent avoir acquis une perception plus positive des femmes qui les entourent. Elles rappellent alors leur réticence initiale à s'engager dans un groupe exclusivement féminin: peur du «placotage », crainte des rivalités entre femmes, méfiance à l'égard du féminisme, etc. Ces femmes se montrent ensuite étonnées d'avoir autant apprécié l'atmosphère du groupe et les échanges de vues, $d^{\prime}$ avoir pu constater la force de l'entraide et, pour certaines d'entre elles, d'avoir créé des relations privilégiées avec une ou plusieurs participantes. Elles n'ont pourtant réalisé, selon l'expression de S. Sturdivant (1980), que "l'apprentissage de la solidarité féminine». On peut sans doute conclure que ces changements dans les attitudes se traduisent bien souvent par l'adoption de comportements nouveaux : nouveaux choix de rôles, nouveaux choix relationnels et parfois engagement plus important dans la communauté en tant que femme.

Pour compléter cette étude des résultats, il apparaît intéressant de mettre en évidence, en s'appuyant cette fois sur une autre typologie, l'impact de facteurs plus difficiles à cerner, soit ceux relatifs à I'influence des thérapeutes. Citons à ce point les résultats d'un projet de recherche de Johnson (1976), du Collectif thérapeutique féministe de Philadelphie, qui stipule que le fait que les thérapeutes féministes soient considérées comme des "modèles de rôles » désirables et puissants est I'un des facteurs de réussite de la thérapie féministe. Dans son compte rendu de recherche, Johnson constate en effet que trois des facteurs jugés les plus utiles par les participantes sont des éléments féministes: perception de la thérapeute comme une personne 
compétente, connaissance du fait qu'en tant que femme elle a partagé l'expérience féminine et, enfin, découverte que les autres femmes sont centrales à leur existence et leur sont d'un grand secours (Sturdivant, 1980 : 108). À I'occasion des bilans de nos groupes, cette notion de relation positive avec les animatrices, celle d'appréciation du climat, de l'ambiance ou encore la découverte d'une complicité avec les autres femmes sont fréquemment rapportées. II nous semble enfin pertinent d'émettre l'hypothèse, liée à la solidarité féminine, que notre modèle de coanimation axé sur la coopération plutôt que sur la compétition a pu avoir un impact positif sur la perception des membres de la possibilité $d^{\prime}$ 'une collaboration réelle et agréable entre femmes.

\section{UNE INTERPRÉTATION DES RÉSULTATS : VERS LA PRÉVENTION SOCIALE}

Notre interprétation des résultats mentionnés nous permet aussi de supposer que notre programme rejoint deux importantes stratégies de prévention sociale en santé mentale, soit celles de l'empowerment et du soutien social.

\section{L'empowerment}

Afin de parvenir à préciser en quoi ce type de groupe peut contribuer à l'empowerment de la clientèle, arrêtons-nous d'abord à une définition de cette notion couramment utilisée en intervention sociale depuis les années 80 , mais plus récemment admise dans le champ de la santé mentale. Selon Swift et Levin (dans Guberman et al., 1993: 121), l'empowerment renvoie à des « activités orientées sur l'augmentation du contrôle des gens sur leur propre vie». Sans aucun doute, notre programme comporte de multiples occasions pour les membres d'acquérir plus de pouvoir sur divers aspects de leur vie: collectivisation des problèmes, apprentissage d'habiletés sociales, entraide, lutte contre I'isolement, etc. Ce type de groupe concerne donc I'appropriation du pouvoir en ce qu'il donne accès à certaines étapes de l'empowerment que sont la conscientisation, l'action personnelle et parfois collective. L'action politique comme telle en est cependant le plus souvent exclue. Certains des principes de pratique relatifs à I'empowerment de Mullender et Ward (1991) sont rejoints, tels la perception de la clientèle comme ayant un potentiel de croissance, la reconnaissance du droit des personnes à être entendues et à avoir 
un pouvoir sur leur vie, y compris sur l'intervention en cours, et, enfin, le reflet par la pratique d'une compréhension globale des multiples facteurs influant sur la situation-problème. Ces principes, bien qu'ils soient formulés différemment, sont d'ailleurs très rapprochés de ceux qu'a définis Walker (1988) pour l'intervention féministe.

Dans le but de mieux cerner les liens de notre type d'intervention avec l'empowerment, arrêtons-nous à considérer la typologie de Whitmore (1990). Celle-ci situe les signes d'empowerment à trois différents plans, soit le plan individuel, le plan du groupe et le plan environnemental. En vue d'évaluer un programme d'éducation prénatale destiné à un groupe de mères célibataires, Whitmore (1990 : 217) est parvenue à identifier des composantes pour les trois aspects mis en évidence. Sur le plan individuel, on y trouve l'amélioration de la confiance en soi, I'acquisition de compétences et d'habiletés et I'apprentissage du plaisir. Par rapport à nos groupes, ces diverses composantes ont clairement été présentées précédemment comme faisant partie des résultats des bilans. Le niveau du groupe chez Whitmore concerne le fait de vaincre l'isolement et le développement d'une confiance plus grande envers les autres. Dans notre intervention, le premier de ces aspects correspond à l'un des changements sur le plan des comportements selon Home (amélioration de la vie sociale). Le second rejoindrait plutôt des résultats constatés sur le plan des attitudes (plus grande confiance envers les autres femmes, en lien avec un changement de perception de celles-ci). Le troisième niveau, axé sur les acquis sur les plans communautaire et politique, apparaît plus éloigné de notre intervention, les aspects susceptibles de $s^{\prime} y$ rapporter étant les actions faites en vue de modifier le vécu professionnel, de même qu'une plus grande sensibilisation au vécu des femmes dans leur milieu de vie. Le but ultime de l'empowerment, qui est de réduire les iniquités de notre système politique et économique en vue de permettre aux personnes de jouer le rôle qui leur revient dans leur communauté de vie, n'est pas directement ciblé, mais les changements constatés aux deux autres niveaux peuvent, croyons-nous, être considérés comme un premier pas dans cette direction. L'opinion de Whitmore (1990: 228) va dans ce sens, à la condition que l'on garde bien présent à l'esprit I'objectif ultime: "The key is not to lose sight of the larger goal...»

\section{Le soutien social}

II convient de signaler, en terminant cette partie sur notre interprétation des résultats, I'accès fourni par notre programme à une autre 
stratégie de prévention en santé mentale, soit celle du soutien social. Partons alors de la définition que lui donne Goettlieb (dans Guberman et al., 1993: 123), à savoir un échange de ressources entre pairs, à l'intérieur de relations préétablies ou d'interventions d'aide planifiées. Quatre formes de transactions seraient couvertes par cette définition: I'expression d'une préoccupation d'ordre émotif, l'aide instrumentale, l'information sur l'environnement et la facilitation de l'auto-évaluation. Notre intervention rejoint ces divers types de transactions soit à l'intérieur des rencontres durant l'échange ou dans les communications plus informelles entre les rencontres ou au moment des pauses. Il importe également de souligner le fait que certaines participantes maintiennent des contacts après la fin des rencontres: il s'agit alors d'amitiés dyadiques ou de petits groupes issus des groupes formels, mais qui ne constituent pas des groupes d'entraide tels que définis dans la littérature en service social.

\section{CONCLUSION}

Nous avons mis en évidence les acquis liés à la forme d'intervention effectuée et nous avons travaillé à préciser les fondements théoriques qui la sous-tendent. Savoir reconnaître les multiples bénéfices engendrés par cette expérience nous paraissait nécessaire, mais il ne faudrait cependant pas perdre de vue certaines limites de l'intervention menée. L'orientation privilégiée, à savoir une intervention de groupe d'inspiration féministe, apparaît nettement justifiée, mais notre interrogation principale porte sur le maintien des changements obtenus. On sait que les recherches sur cet aspect concluent souvent à l'évidence de résultats pessimistes et qu'elles insistent sur la primauté du rôle de l'environnement de la personne pour favoriser le maintien des acquis (Rondeau, 1988). La question du maintien des acquis en intervention clinique demeure malheureusement peu connue, peu inventoriée et les résultats précédemment mentionnés n'abordent pas ce problème malgré le souci omniprésent véhiculé sur ce plan dans nos modalités d'intervention. Une des stratégies les plus plausibles de maximisation du soutien social ne résiderait-elle pas dans la création d'un groupe d'entraide formé de membres des divers groupes et pour lequel les animatrices deviendraient au besoin consultantes? 


\section{Références bibliographiques}

BeAuvolR, S. de (1949). Le deuxième sexe. Paris: Gallimard, Coll. Idées, tome 2.

BROCHU, R. et M. BROUSSEAU-GINGRAS (1984). Le service social psychiatrique au CHUL. Identification de la clientèle et propositions de changements, Service social hospitalier, CHUL. Document.

Broverman, I., O. Broverman, F. Clarkson, P. Rosenkrantz et S. Vogel (1970). "Sex Role Stereotypes and Clinical Judgments of Mental Health », Journal of Consulting and Clinical Psychologists, $\mathrm{n}^{\circ} 34: 1-7$.

CARDin, M. et A. HOME (1983). "La pratique du service social avec les groupes de femmes", Service social, , vol. 32, no 1-2: 170-186.

COOPERSTOCK, R. (1980). "Problèmes particuliers liés à I'usage des psychotropes chez les femmes", Santé mentale au Canada, vol. 28, n 2.

Corbeil, C., A. Paquet-Deehy, C. Lazure et G. Legault (1983). L'intervention féministe, l'alternative au sexisme en thérapie. Montréal: Éd. coop. A. Saint-Martin.

Gilligan, C. (1982). In a Different Voice: Psychological Theory and Women's Development. Cambridge: Harvard University Press.

Guberman, N., J. LindSAy, L. SPeCtOr et al. (1993). Le défi de l'égalité. La santé mentale des hommes et des femmes. Le Comité de la santé mentale du Québec. Boucherville: Éditions Gaëtan Morin.

HOME, A. (1988). "Les groupes de femmes: outils de changement personnel et de développement», Service social, vol. 37, n 1-2:61-86.

JOHNSON, M. (1976). "An Approach to Feminist Therapy», Psychotherapy: Theory, Research and Practice, vol. 13, $\mathrm{n}^{\circ} 1: 72-76$.

Mullender, A. et P. WARD (1991). Self-Directed Groupwork. Londres: Whiting and Birch.

RONDEAU, S. (1988). Le maintien et le transfert du changement en travail de groupe. Essai de maîtrise. Sainte-Foy, Université Laval.

RUSSEL, M. (1984). Skills in Counseling Women : the Feminist Approach. Springfield: Thomas.

SHERMAN, J., C. KOUfACOS et J.A. KENWORTHY (1978). "Therapists: their Attitudes and Information about Women», Psychology of Women Quarterly, vol. 2, $\mathrm{n}^{\circ} 4$.

StURDIVANT, S. (1980). Les femmes et la psychothérapie, Psychologie et sciences humaines. Bruxelles: Mardaga.

Walker, A. (1988). "Feminist Programs for Families», Family Relations, vol. $37: 17-22$.

Whitmore, E. (1990). "Empowerment in Program Evaluation, a Case Example», Revue canadienne de service social, vol. 7, n 2 : 215-230.

WOOLF, V. (1929). Une chambre à soi. Paris: Denoël-Gonthier, Coll. Femmes. WyCKOff, H. (1980). Solving Problems Together. New York: Grove Press. 